\title{
Gap solitons attached to a gapless layer
}

\author{
Thawatchai Mayteevarunyoo ${ }^{1, *}$ and Boris A. Malomed ${ }^{2}$ \\ ${ }^{1}$ Department of Telecommunication Engineering, Mahanakorn University of Technology, Bangkok 10530, Thailand \\ ${ }^{2}$ Department of Physical Electronics, School of Electrical Engineering, \\ Faculty of Engineering, Tel Aviv University, Tel Aviv 69978, Israel
}

compiled: May 14, 2021

\begin{abstract}
We consider linear and nonlinear modes pinned to a grating-free (gapless) layer placed between two symmetric or asymmetric semi-infinite Bragg gratings (BGs), with a possible phase shift between them, in a medium with the uniform Kerr nonlinearity. The asymmetry is defined by a difference between bandgap widths in the two BGs. In the linear system, exact defect modes (DMs) are found. Composite gap solitons pinned to the central layer are found too, in analytical and numerical forms, in the nonlinear model. In the asymmetric system, existence boundaries for the DMs and gap solitons, due to the competition between attraction to the gapless layer and repulsion from the reflectivity step, are obtained analytically. Stability boundaries for solitons in the asymmetric system are identified by means of direct simulations. Collisions of moving BG solitons with the gapless layer are studied too.
\end{abstract}

OCIS codes: (190.5530) Pulse propagation and temporal solitons; (160.5293) Photonic bandgap materials; (060.3735) Fiber Bragg gratings.

http://dx.doi.org/10.1364/XX.99.099999

* Corresponding author: thawatch@mut.ac.th 


\section{Introduction and the model}

Many optical media are based on Bragg gratings (BGs), which are built as modulations of the local refractive index with the spatial period close to the half-wavelength of the electromagnetic field. Fiber BGs, which operate in the temporal domain, are used in numerous applications, and are also a subject of fundamental studies [1, 2]. The interplay of the BG with the nonlinearity of the fiber material gives rise to gap solitons $[3]-[5]$, which were originally predicted as analytical solutions [6]-[9], and then created in experiments [10]-[16]. Spatial BG solitons were predicted too, using periodic gratings written on a planar waveguide [17]-[19] or in a photonic crystal [20]. Solitons can also be supported by a combination of the BG with the second-harmonic-generating [21]-[24] or resonant (two-level) 225, 26] nonlinearity.

The variety of modes supported by the BGs and their potential applications can be expanded by using various superstructures created on top of regular gratings. The technology of the creation of such complex gratings in optical fibers became available long ago [27]-[29]. In particular, supergratings give rise to additional spectral bandgaps, in addition to the central one induced by the underlying uniform BG [30]. Theoretical models of the supergratings, incorporating the nonlinearity of the material, were developed in detail [30]-[32].

Another example of the superstructure is known in the form of the Moiré pattern, with a sinusoidal modulation imposed on the periodic variation of the refractive index building the ordinary BG. It gives rise to a narrow transmission band in the middle of the central gap (resembling the effect of the electromagnetically-induced transparency), which was proposed [33, 34] and implemented experimentally [35] for the retardation of light in the BG.

It is also possible to design BG superstructures patterns in a "semi-discrete" form, i.e., as a uniform nonlinear waveguide with periodically inserted narrow Bragg reflectors [36], and as a more general scheme, which makes use of a periodic modulation of the BG chirp and local Bragg reflectivity [37]. On the other hand, complex grating patterns can be readily written in photonic crystals and photonic-crystal fibers [38 40].

Another relevant setting is a uniform nonlinear BG with one or several local defects (rather than defect lattices) [41]-45]. In particular, solitons pinned to a defect represented by a $\delta$-functional perturbation of the local refractive index or Bragg reflectivity can be found in an exact analytical form [42]. The delta-function approximates a BG with a narrow embedded layer carrying large reflectivity of the opposite sign. Interesting results are also produced by models which include symmetric pairs of defects: repulsive ones form cavities trapping solitons which perform shuttle oscillations, while attractive pairs give rise to pinned states featuring spontaneous symmetry breaking [47]. The interaction of moving BG solitons with weak local defects can be described by means of the perturbation theory which was developed in detail in Refs. [48]-[50].

The nonlinear light propagation in the "apodized" BG, i.e., one with a spatially inhomogeneous Bragg reflectivity, obeys the system of coupled-mode equations for amplitudes $u$ and $v$ of the right- and left-traveling waves [31, 48 [50]:

$$
\begin{gathered}
i \frac{\partial u}{\partial t}+i \frac{\partial u}{\partial x}+\kappa(x) v+\left(|v|^{2}+\frac{1}{2}|u|^{2}\right) u=0, \\
i \frac{\partial v}{\partial t}-i \frac{\partial v}{\partial x}+\kappa^{*}(x) u+\left(|u|^{2}+\frac{1}{2}|v|^{2}\right) v=0,
\end{gathered}
$$

where $t$ and $x$ are the time and coordinate normalized so that the group velocities of the waves are \pm 1 , ratio $2: 1$ of the cross- and self-phase-modulation coefficients in the cubic terms is a standard feature of the Kerr effect [5], and $\kappa(x)$ is the inhomogeneous Bragg reflectivity, which may be complex, taking into regard a phase shift between the incident and reflected waves (the asterisk stands for the complex conjugate). Replacing $t$ by propagation distance $z$, the same coupled-mode system may be realized in the spatial domain, i.e., for planar waveguides instead of fibers.

A local finite-width defect corresponding to

$$
\kappa(x)=1-b \operatorname{sech}(k x),
$$

which describes a local depression of the Bragg reflectivity, was studied in Ref. [44]. Because such a defect attracts the wave fields, it can support localized pinned modes, including defect modes (DMs) in the linearized version of the model. The DMs and their nonlinear extension were constructed in Ref. [44] by means of numerical methods, and the stability of the nonlinear pinned modes was investigated. It was found that the instability is caused by resonances of perturbations with the continuous spectrum.

The aim of the present work is to introduce a similar but simpler and, in a certain sense, more general, configuration, with a gapless layer placed between two semi-infinite gratings. We define two versions of this system. In one, the 
semi-infinite BGs are mutually symmetric, with a possible phase shift $(\alpha)$ between them:

$$
\kappa(x)=\left\{\begin{array}{c}
1, \text { at } x>0, \\
0, \text { at }-L<x<0, \\
\exp (i \alpha), \text { at } x<-L
\end{array}\right.
$$

where $|\kappa|=1$ for $\kappa \neq 0$ may be fixed by obvious rescaling, provided that reflectivity $|\kappa|$ takes equal absolute values in both semi-infinite gratings. The second system is composed of asymmetric BGs, with unequal reflectivities, $\kappa=1$ and $\kappa=\cos \alpha$ (here we set $0 \leq \alpha<\pi / 2$ ):

$$
\kappa(x)=\left\{\begin{array}{c}
1, \text { at } x>0, \\
0, \text { at }-L<x<0, \\
\cos \alpha, \text { at } x<-L .
\end{array}\right.
$$

The presence of parameter $\alpha$ in either version, based on Eq. (3) or (4), is an essential generalization in comparison with the model based on Eq. (2). A still more general configuration, with a phase shift between asymmetric BGs, is possible too, but such system with the double complexity is beyond the scope of the present paper. Another advantage of the defects with the simple shapes defined by Eqs. (3) and (4) is that it is much easier to realize them in the experiment. Further, we demonstrate below that both linear DMs and gap solitons pinned by the gapless layer can be found in an analytical form, which is not possible in the case of the more sophisticated profile (2). In particular, we conclude that the nonlinear extension of the DMs fully merges into families of the pinned solitons.

It is necessary to mention that Eq. (11) neglects the ordinary dispersion or diffraction in the gapless layer, in the temporal and spatial implementations of the BG, respectively. This assumption is relevant if the respective dispersion/diffraction length, corresponding to $L$, is much larger than the characteristic soliton-formation length, $z_{\text {sol }}$. Note that, in physical units, $L=1$ (this scale plays a dominant role below) corresponds to length $\sim 1 \mathrm{~mm}$, in the spatial and temporal domains alike, and, in either case, $z_{\text {sol }}$ takes values between $1 \mathrm{~mm}$ and $1 \mathrm{~cm} \mathrm{[1]-[3],} \mathrm{[17,} \mathrm{18].}$ With the carrier wavelength $\simeq 1.5 \mu \mathrm{m}, L \sim 1 \mathrm{~mm}$ corresponds to diffraction length $\sim 10 \mathrm{~cm} \gg z_{\text {sol }}$. In the temporal domain, the disparity between the characteristic dispersion length and $z_{\text {sol }}$ is sill larger.

The diffraction in the gapless region should be taken into regard in the limit of $L \rightarrow \infty$, which actually corresponds to an interface (surface) separating a semi-infinite BG and the uniform medium. The interaction of gap surface solitons with such an interface was studied before [13]-[15].

The subsequent presentation is structured as follows. Analytical results are presented in Section II. They include exact solutions for linear DMs in both models (3i) and (4), a perturbative treatment of its weakly nonlinear extension (explicitly presented for the symmetric system, with $\alpha=0$ ), which is well corroborated by numerical results, and an implicit analytical solution for solitons pinned to the gapless layer. Section III reports basic numerical results for the nonlinear asymmetric system, based on Eq. (4), including its symmetric limit, with $\alpha=0$. The numerical results display existence and stability areas for the pinned solitons in the underlying parameter space, and simulations of collisions of moving solitons with the gapless layer (for another type of the defect, collisions were simulated in Refs. [41] and [42]). In particular, the collision may generate a trapped soliton from a part of the energy of the incident one, which can be used for the creation of standing optical solitons (thus far, the best experimental result for slow-light BG solitons was the creation of one moving at velocity $\approx 0.16$ speed of light in vacuum [16]).

\section{Analytical results}

\section{A. Stationary equations}

Stationary solutions of Eq. (11) with frequency $\omega$ are looked for as

$$
\{u(x, t), v(x, t)\}=e^{-i \omega t}\{U(x), V(x)\},
$$

where complex functions $U$ and $V$ satisfy the system of ordinary differential equations:

$$
\begin{aligned}
& +i \frac{d U}{d x}+\omega U+\kappa(x) V+\left[\left(|V|^{2}+\frac{1}{2}|U|^{2}\right)\right] U=0, \\
& -i \frac{d V}{d x}+\omega V+\kappa^{*}(x) U+\left[\left(|U|^{2}+\frac{1}{2}|V|^{2}\right)\right] V=0,
\end{aligned}
$$

which are compatible with the constraint reducing the two stationary fields to one,

$$
U^{*}(x)=-V(x) .
$$


The stationary solutions are characterized by the total energy, alias norm (or total power, in terms of the spatialdomain model) and Hamiltonian,

$$
\begin{gathered}
E=\int_{-\infty}^{+\infty}\left[|U(x)|^{2}+|V(x)|^{2}\right] d x \\
H=\int_{-\infty}^{+\infty}\left\{\left[\frac{i}{2}\left(U \frac{d U^{*}}{d x}-V \frac{d V^{*}}{d x}\right)+\text { c.c. }\right]-\frac{1}{4}\left(|U|^{4}+|V|^{4}+|U|^{2}|V|^{2}\right)\right\} d x+H_{\text {Bragg }} \\
H_{\text {Bragg }}=-\int_{-\infty}^{+\infty}\left[\kappa(x) U^{*} V+\text { c.c. }\right] d x,
\end{gathered}
$$

where c.c., as well as $*$, stand for the complex conjugate.

The well-known analytical solution for the quiescent BG solitons in the uniform grating, with $L=0$ and constant $\kappa \equiv|\kappa| e^{i \alpha}$, corresponds to general expressions (5) and (17), with [6, 8, 9]

$$
\begin{gathered}
U_{\mathrm{sol}}=\sqrt{\frac{2}{3}|\kappa|} \mid e^{i \alpha / 2} \frac{\sin \theta}{\cosh (|\kappa| x \sin \theta-i \theta / 2)} \\
\omega_{\mathrm{sol}}=|\kappa| \cos \theta
\end{gathered}
$$

Here, $\theta$ is an intrinsic parameter of the soliton family which takes values $0<\theta<\pi$, with the respective soliton's frequency (12) belonging to the bandgap of the linearized version of Eqs. (11),

$$
-|\kappa|<\omega<+|\kappa|
$$

The total energy (8) of this exact solution is

$$
E_{\mathrm{sol}}=(8 / 3) \theta
$$

(note that $E_{\text {sol }}$ does not explicitly depend on $|\kappa|$ ). It is known that the exact solitons are stable, in the uniform medium, at [52]-[54], 24]

$$
\theta \leq \theta_{\max } \approx 1.01 \times \pi / 2
$$

i.e., in the interval of frequencies

$$
\left(\omega_{\mathrm{sol}}\right)_{\mathrm{cr}} \approx-0.016 \leq \omega_{\mathrm{sol}} \leq 1
$$

cf. the full bandgap (13).

In the asymmetric system with reflectivity profile (4), the full bandgap is reduced, per Eq. (13), from $\omega^{2}<1$ to $\omega^{2}<\cos ^{2} \alpha$. In the interval between the bandgaps of the two semi-infinite gratings, i.e., at

$$
\cos ^{2} \alpha<\omega^{2}<1
$$

the asymmetric system gives rise to states which are localized at $x>0$ but delocalized at $x<-L$, see below.

Exact solution (11) features an obvious symmetry, $\left\{U_{\text {sol }}(-x), V_{\text {sol }}(-x)\right\}=\left\{U_{\text {sol }}^{*}(x), V_{\text {sol }}^{*}(x)\right\}$. Similarly, localized modes in the system based on Eqs. (11) and (3) with $\alpha=0$ are symmetric with respect to the midpoint of the gapless layer, $x=-L / 2$ :

$$
\{U(-x), V(-x)\}=\left\{U^{*}(x-L), V^{*}(x-L)\right\}
$$

The presence of the phase shift $(\alpha)$ in Eq. (3) between the semi-infinite gratings affects this relation (see below), but the corresponding intensity profile remains symmetric:

$$
|U(-x)|^{2}+|V(-x)|^{2}=|U(x-L)|^{2}+|V(x-L)|^{2}
$$




\section{B. The linear defect mode}

\section{B.1. The symmetric system}

The linearized version of Eq. (6), subject to reduction (7), reduces to the single linear equation,

$$
i \frac{d U}{d x}+\omega U-\kappa(x) U^{*}=0 .
$$

This equation, with $\kappa(x)$ taken as per Eq. (3) for the symmetric system, including the phase shift, gives rise to an exact composite (three-layer) DM solution with eigenfrequency $\omega_{\text {eigen }}$ :

$$
U(x) \equiv \varepsilon U_{1}(x)=\varepsilon\left\{\begin{array}{c}
\exp \left[\frac{i}{2} \cos ^{-1}\left(\omega_{\text {eigen }}\right)-\sqrt{1-\omega_{\text {eigen }}^{2}} x\right], \text { at } x \geq 0, \\
B_{\mathrm{DM}} \exp \left(i \omega_{\text {eigen }}\left(x+\frac{L}{2}\right)\right) \text {, at }-L \leq x \leq 0, \\
\exp \left[\frac{i}{2} \alpha-\frac{i}{2} \cos ^{-1}\left(\omega_{\text {eigen }}\right)+\sqrt{1-\omega_{\text {eigen }}^{2}}(x+L)\right], \text { at } x \leq-L,
\end{array}\right.
$$

where $\varepsilon$ is an infinitesimal amplitude which underlies the linearization. Relative amplitude $B_{\mathrm{DM}}$ and the eigenfrequency [it always falls into bandgap (13)] are determined by the condition of the continuity of $U(x)$ at $x=0$ and $x=-L$ :

$$
\begin{gathered}
B_{\mathrm{DM}}=\exp (i \alpha / 4+i \pi n), \\
\omega_{\text {eigen }} L+(\alpha / 2)=\cos ^{-1}\left(\omega_{\text {eigen }}\right)+2 \pi n,
\end{gathered}
$$

where $n$ is an arbitrary integer. Note that the DM given by Eq. (21) is fully symmetric about the midpoint, as per definition (18), at $\alpha=0$, and satisfies symmetry relation (19) at any $\alpha$. The respective integral energy (8) is $E_{\mathrm{DM}}=\varepsilon^{2}\left(L+1 / \sqrt{1-\omega_{\text {eigen }}^{2}}\right)$.

For a weak gapless layer, i.e., $L \rightarrow 0$ and $\alpha \rightarrow 0$, Eq. (23) for the fundamental DM (with $n=0$ ) yields

$$
\omega_{\text {eigen }} \approx 1-(1 / 8)(\alpha+2 L)^{2},
$$

with solution (21) degenerating into a constant at $L=\alpha=0$. On the other hand, at $\alpha \rightarrow \pi$, Eq. (23) with $n=0$ yields a vanishingly small eigenfrequency,

$$
\omega_{\text {eigen }} \approx \frac{1}{2} \frac{\pi-\alpha}{1+L}
$$

Further, the largest possible eigenfrequency, $\omega_{\text {eigen }}=1$, is attained by the solution to Eq. (23) for the fundamental $\mathrm{DM}(n=0)$ at $\alpha\left(\omega_{\text {eigen }}=1\right)=-2 L$, and close to this point the solution takes the same form as given by Eq. (24). The fundamental DM does not exist at

$$
\alpha<\alpha_{\min } \equiv-2 L
$$

The relation between the eigenfrequency and $\alpha$, produced by Eq. (23) with $n=0$, is displayed, for $L=0.5$ and $L=2.0$, in Fig. $10\left[\omega<0\right.$ corresponds to the DM subject to constraint $U(x)=+V^{*}(x)$, instead of Eq. (77)], which demonstrates that the relation keeps its qualitative shape at all relevant values of the gapless-layer's width. In particular, in the limit of $L \rightarrow 0$, which corresponds to the junction of two semi-infinite gratings with phase jump $\alpha$ between them (here, we can fix $0 \leq \alpha \leq \pi$ ), straightforward consideration of Eq. (23) yields

$$
\omega_{\text {eigen }}(L \rightarrow 0)=\cos (\alpha / 2)-(L / 2) \sin \alpha+O\left(L^{2}\right),
$$

the expansion of which at $\alpha \rightarrow 0$ complies with Eq. (24). At $\alpha=0$, characteristic eigenfrequencies are

$$
\omega_{\text {eigen }}(L=0.5) \approx 0.90, \omega_{\text {eigen }}(L=1.0) \approx 0.74, \omega_{\text {eigen }}(L=1.5) \approx 0.61, \omega_{\text {eigen }}(L=2.0) \approx 0.51 .
$$

At large values of $L$, higher-order DMs appear, corresponding to $n>0$ in Eq. (23) [higher-order linear DMs were also found numerically in the model including the defect profile (2) [44]]. Indeed, higher-order DMs emerge, for each $n \geq 1$, at $\omega=1$, i.e., at

$$
L_{\mathrm{cr}}^{(n)}=2 \pi n-\alpha / 2 .
$$

For instance, at $\alpha=0$ and with $L$ slightly exceeding the first critical value (29), i.e., $L=2 \pi+l, 0<l \ll 2 \pi$, the emerging eigenfrequency is obtained as $\omega_{\text {eigen }}^{(n=1)} \approx 1-l^{2} / 2$, cf. Eq. (24), while its fundamental counterpart, found as 


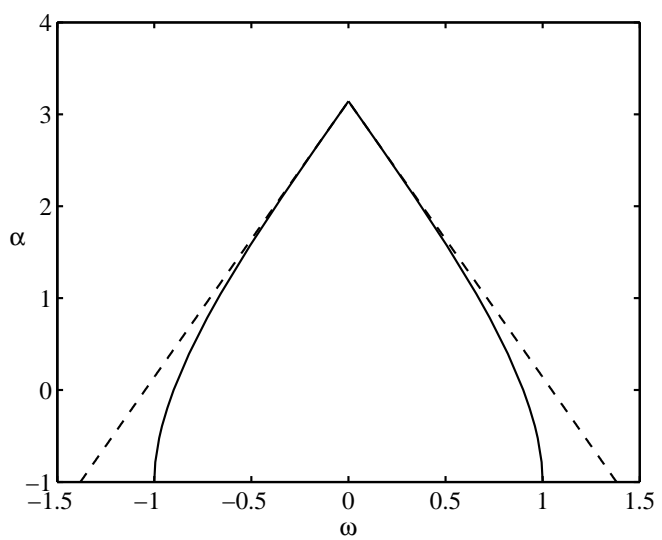

(a)

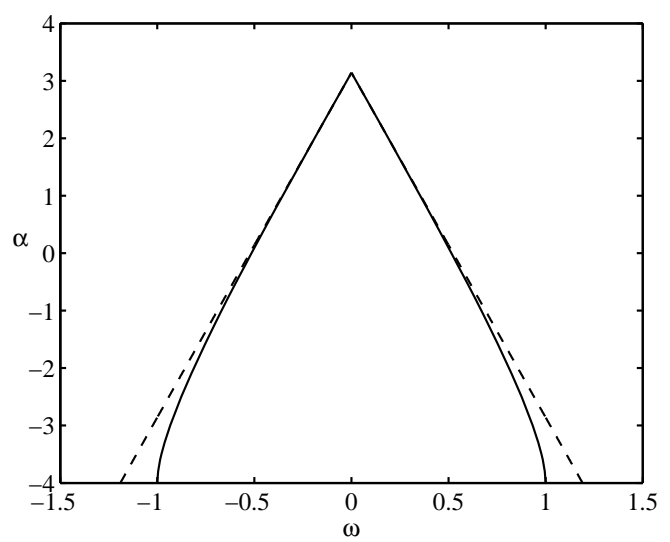

(b)

Fig. 1. Relations between the eigenfrequency of the fundamental defect mode, in the linear symmetric system with phase shift $\alpha$ [defined per Eq. (3)], and $\alpha$, as produced by Eq. [23) with $n=0$, at fixed values of the width of the gapless layer: (a) $L=0.5$; (b) $L=2.0$. The dashed straight lines depict analytical approximation (25).

a numerical solution of Eq. (23), is $\omega_{\text {eigen }}^{(n=0)}(L=2 \pi) \approx 0.22$. As follows from Eq. (29), for given $L$ the total number of defect modes is

$$
n_{\mathrm{DM}}=1+\left[\frac{L+(\alpha / 2)}{2 \pi}\right],
$$

where $[. .$.$] denotes the integer part.$

Below, reporting numerical results for the full nonlinear system based on Eqs. (11) and (3), we focus on moderately wide gapless layers, with $L \leq 2$, and $0 \leq \alpha \leq \pi / 3$, for which there is the single DM, corresponding to $n=0$.

Outside of bandgap (13), an exact composite solution of the linearized equation (20) for delocalized states can be found too [cf. Eq. (21)]:

$$
U(x)=\varepsilon\left\{\begin{array}{c}
A_{+} \exp \left(i \sqrt{\omega^{2}-1} x\right)+A_{-} \exp \left(-i \sqrt{\omega^{2}-1} x\right), \text { at } x \geq 0, \\
\exp \left(i \omega\left(x+\frac{L}{2}\right)\right), \text { at }-L \leq x \leq 0, \\
C_{+} \exp \left(i \sqrt{\omega^{2}-1}(x+L)\right)+C_{-} \exp \left(-i \sqrt{\omega^{2}-1}(x+L)\right), \text { at } x \leq-L,
\end{array}\right.
$$

where the amplitudes are

$$
\begin{aligned}
& A_{+}=\frac{e^{\frac{i}{2} \omega L}-e^{-\frac{i}{2} \omega L}\left(\omega-\sqrt{\omega^{2}-1}\right)}{2 \sqrt{\omega^{2}-1}\left(\omega+\sqrt{\omega^{2}-1}\right)}, A_{-}=\left(\omega-\sqrt{\omega^{2}-1}\right) A_{+}^{*}, \\
& C_{+}=\frac{e^{-\frac{i}{2} \omega L}-e^{\frac{i}{2} \omega L+i \alpha}\left(\omega-\sqrt{\omega^{2}-1}\right)}{2 \sqrt{\omega^{2}-1}\left(\omega+\sqrt{\omega^{2}-1}\right)}, C_{-}=\left(\omega-\sqrt{\omega^{2}-1}\right) e^{i \alpha} C_{+}^{*} .
\end{aligned}
$$

The existence of this solution at all $\omega^{2} \geq 1$ corroborates that the insertion of the symmetric gapless layer does not make bandgap (13) broader than in the uniform grating, including the case when the phase shift is present, $\alpha \neq 0$.

\section{B.2. The asymmetric system}

The exact three-layer solution (21)-(23) for the linear DM can be easily generalized for the system with asymmetric reflectivity profile (4):

$$
\begin{gathered}
U(x) \equiv \varepsilon U_{1}(x)=\varepsilon\left\{\begin{array}{c}
\exp \left[\frac{i}{2} \cos ^{-1}\left(\omega_{\text {eigen }}\right)-\sqrt{1-\omega_{\text {eigen }}^{2}} x\right], \text { at } x \geq 0, \\
\tilde{B}_{\mathrm{DM}} \exp \left(i \omega_{\text {eigen }}\left(x+\frac{L}{2}\right)\right), \text { at }-L \leq x \leq 0, \\
\exp \left[-\frac{i}{2} \cos ^{-1}\left(\frac{\omega_{\text {eigen }}}{\cos \alpha}\right)+\sqrt{\cos ^{2} \alpha-\left(\omega_{\text {eigen }}\right)^{2}}(x+L)\right], \text { at } x \leq-L,
\end{array}\right. \\
\tilde{B}_{\mathrm{DM}}=\exp \left[\frac{i}{2}\left(\cos ^{-1}\left(\omega_{\text {eigen }}\right)-\omega_{\text {eigen }} L\right)\right],
\end{gathered}
$$


and Eq. (23) for the eigenfrequency is replaced by

$$
\omega_{\text {eigen }} L=\frac{1}{2}\left[\cos ^{-1}\left(\omega_{\text {eigen }}\right)+\cos ^{-1}\left(\frac{\omega_{\text {eigen }}}{\cos \alpha}\right)\right]+2 \pi n \text {. }
$$

In the symmetric system, the DM exists at all values of width $L$ of the gapless layer, including $L=0$ [see Eq. [27] ], provided that the phase shift satisfies condition $\alpha>-2 L$, see Eq. (26). The situation is drastically different in the asymmetric system, because, while the gapless layer attracts the DM (or soliton, see below), the reflectivity step, accounted for by $\cos \alpha<1$ in Eq. (4), repels the localized mode. The result of the competition of the attraction and repulsion is that, for given $\alpha$, a solution of Eq. (35) exists only if $L$ exceeds a minimum value,

$$
L \geq L_{\min }^{(\mathrm{DM})}=\frac{\alpha+4 \pi n}{2 \cos \alpha},
$$

while at $L<L_{\min }$ the DM does not exist. Exactly at $L=L_{\min }$, the eigenfrequency coincides with the edge of the respective bandgap (13), $\omega_{\text {eigen }}=\cos \alpha$. Further, it follows from Eq. (36) that the total number of DMs in the asymmetric system is

$$
\tilde{n}_{\mathrm{DM}}=\left[1+\frac{2 L \cos \alpha-\alpha}{4 \pi}\right]
$$

cf. the similar result (30) for the symmetric model. Another corollary of Eq. (36) is that, for fixed $L$, the fundamental DM (the one with $n=0$ ) in the asymmetric system exists in interval

$$
0 \leq \alpha \leq \alpha_{\max }
$$

with $\alpha_{\max }$ defined by equation

$$
2 L \cos \left(\alpha_{\max }\right)=\alpha_{\max }
$$

At small and large $L$, solutions to Eq. (38) are

$$
\alpha_{\max } \approx\left\{\begin{array}{c}
2 L-4 L^{3}, \text { at } L \rightarrow 0, \\
(\pi / 2)[1-1 /(2 L)], \text { at } L \rightarrow \infty .
\end{array}\right.
$$

Particular solutions are

$$
\alpha_{\max }(L=0.5) \approx 0.74, \alpha_{\max }(L=1.0) \approx 1.03, \alpha_{\max }(L=1.5) \approx 1.17, \alpha_{\max }(L=2.0) \approx 1.25
$$

Finally, for given $L$, the DM's eigenfrequency, $\omega_{\text {eigen, }}$ decreases from its value in the symmetric system [the one determined by Eq. (23) with $\alpha=0$ ] to the above-mentioned value, $\cos \left(\alpha_{\max }(L)\right)$, with the increase of the asymmetry parameter, $\alpha$, from zero to $\alpha_{\max }(L)$. These dependences are displayed in Fig. 2, which can be compared to their counterparts in the symmetric system with phase shift $\alpha$, shown in Fig. 1 ,

As mentioned above, in interval (17) between the broad and reduced bandgaps the asymmetric system supports semi-delocalized linear modes. The explicit form of these composite solutions is

$$
U(x)=\varepsilon\left\{\begin{array}{c}
\exp \left(\frac{i}{2} \cos ^{-1} \omega-\sqrt{1-\omega^{2}} x\right), \text { at } x \geq 0 \\
\exp \left[i\left(\frac{1}{2} \cos ^{-1} \omega+\omega x\right)\right], \text { at }-L \leq x \leq 0, \\
\tilde{C}_{+} \exp \left(i \sqrt{\omega^{2}-\cos ^{2} \alpha}(x+L)\right)+\tilde{C}_{-} \exp \left(-i \sqrt{\omega^{2}-\cos ^{2} \alpha}(x+L)\right), \text { at } x \leq-L,
\end{array}\right.
$$

where the amplitudes are

$$
\begin{aligned}
\tilde{C}_{+} & =\frac{\left(\omega+\sqrt{\omega^{2}-\cos ^{2} \alpha}\right) e^{i \phi}-(\cos \alpha) e^{-i \phi}}{\sqrt{\omega^{2}-\cos ^{2} \alpha}}, \tilde{C}_{-}=\frac{\omega-\sqrt{\omega^{2}-\cos ^{2} \alpha}}{\cos \alpha} C_{+}^{*}, \\
\phi & \equiv \frac{1}{2} \cos ^{-1} \omega-L \omega .
\end{aligned}
$$

cf. Eqs. (31), (32) and (33). 
Fig. 2. (Color online) The relation between the eigenvalue of the fundamental linear defect mode in the asymmetric system and asymmetry parameter $\alpha$, as per Eq. (35) with $n=0$, at fixed values of width $L$ of the gapless layer: $L=0.5, L=1.0$, $L=1.5$, and $L=2.0$. The eigenvalues are shown in their existence region, $0 \leq \alpha \leq \alpha_{\max }(L)$, see the text. The dashed green curve shows the boundary of bandgap (13), $\omega=\cos \alpha$, in the same region.

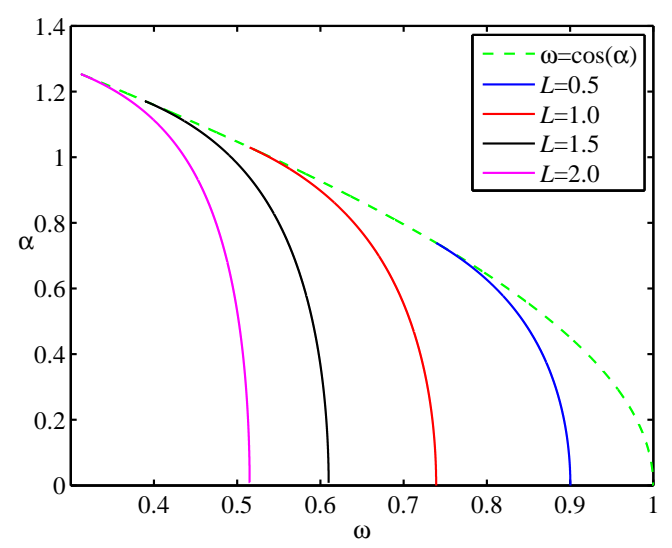

\section{C. The nonlinear correction to the eigenfrequency of the defect mode}

If the weak nonlinearity is taken into account, assuming that $\varepsilon$ in Eqs. (21) and (21) is small but finite, in the first approximation it gives a small correction to the eigenfrequency,

$$
\omega_{\text {eigen }}=\omega_{\text {eigen }}^{(0)}+\varepsilon^{2} \omega_{\text {eigen }}^{(2)} .
$$

We perform further analysis of this situation for the symmetric system, with $\alpha=0$. Multiplying the first equation of system (6) by solution (21), found in the linear approximation, and performing integration over $d x$ yields, with regard to relation (7),

$$
\omega_{\text {eigen }}^{(2)}=-\frac{3}{2} \frac{\int_{-\infty}^{+\infty}\left|U_{1}(x)\right|^{4} d x}{\int_{-\infty}^{+\infty}\left|U_{1}(x)\right|^{2} d x}=-\frac{3}{2} \frac{1+2 \sqrt{1-\left(\omega_{\text {eigen }}^{(0)}\right)^{2}} L}{1+\sqrt{1-\left(\omega_{\text {eigen }}^{(0)}\right)^{2}} L}
$$

Here and in the subsequent numerical analysis, we used the Newton's iteration method to construct nonlinear DMs, using the linear modes, given by Eqs. (21) - (23), as the initial guess. The solution domain, with periodic boundary conditions, is $|x| \leq 60$ (in all the cases, the width of localized modes is much smaller than the size of the domain). The resulting $\omega_{\text {eigen }}(\varepsilon)$ curves for the nonlinear mode is plotted in Fig. 3 for two fixed values of $L$. The figure shows close agreement between the numerically found eigenfrequency and the analytical prediction produced by Eqs. (41) and (42) (the agreement is equally good at other values of $L$ ).

Typical examples of the nonlinear DM, numerically found for $\omega>0$ and $\omega<0$, are shown in Figs. $4(a, b, c)$. As demonstrated below [see Fig. 7(a)], the nonlinear states, i.e., as a matter of fact, pinned gap solitons, displayed here

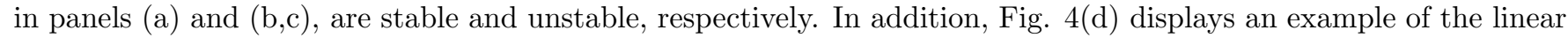
DM.

\section{D. Gap solitons}

\section{D.1. The symmetric system}

Stationary solutions of the nonlinear symmetric system (6), including phase shift $\alpha$, for gap solitons pinned to the gapless layer, which are subject to restriction (7), can also be constructed as composite modes, by juxtaposing solutions (11) in the semi-infinite gratings, and a plane-wave state in the gapless layer [cf. solution (21) for the DM]:

$$
U(x)=\left\{\begin{array}{c}
\sqrt{\frac{2}{3}} \frac{\sin \theta}{\cosh [(\sin \theta)(x+\xi)-i \theta / 2]} \\
B_{\mathrm{sol}} \exp \left[i\left(\omega+\frac{3}{2}\left|B_{\mathrm{sol}}\right|^{2}\right)(x+L)\right] \\
\sqrt{\frac{2}{3}} e^{i \alpha / 2} \frac{\sin \theta}{\cosh [(\sin \theta)(x+L-\xi)-i \theta / 2]}
\end{array}\right.
$$




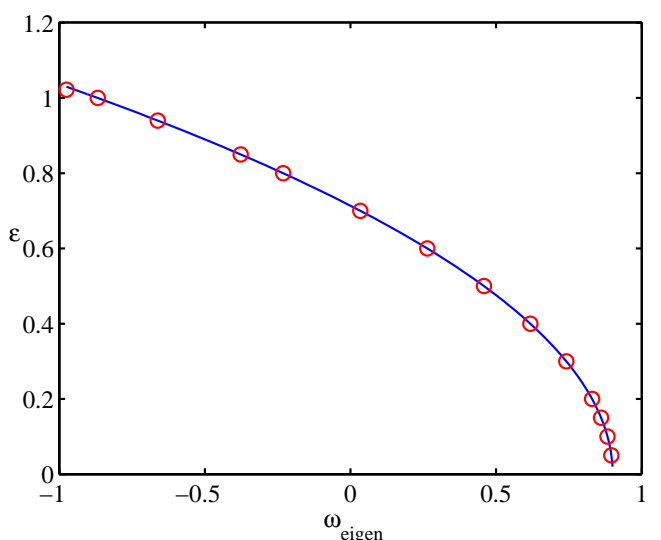

(a)

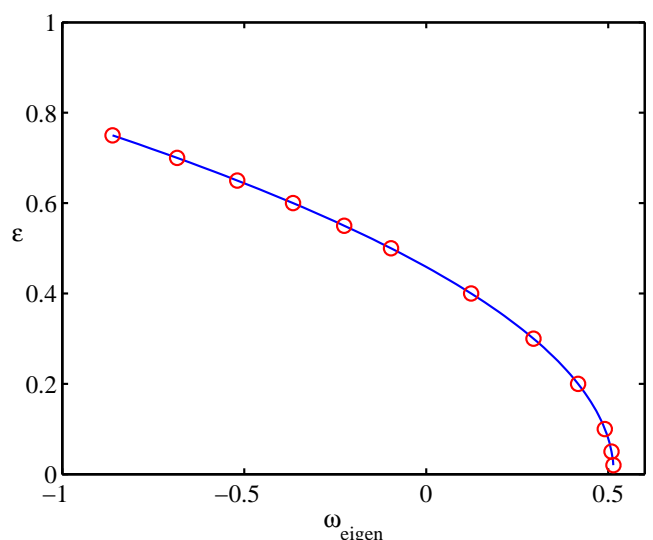

(b)

Fig. 3. (Color online) Chains of circles display numerically found eigenfrequencies of the nonlinear defect mode versus its amplitude in the symmetric system with $\alpha=0$, at different values of the width of the gapless layer: (a) $L=0.5$; (b) $L=2.0$. Blue solid lines represent the respective analytical approximation produced by Eqs. (41) and (42).

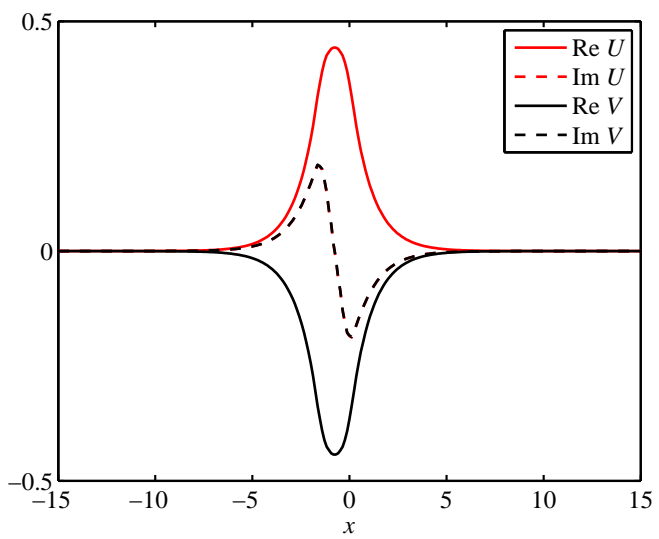

(a)

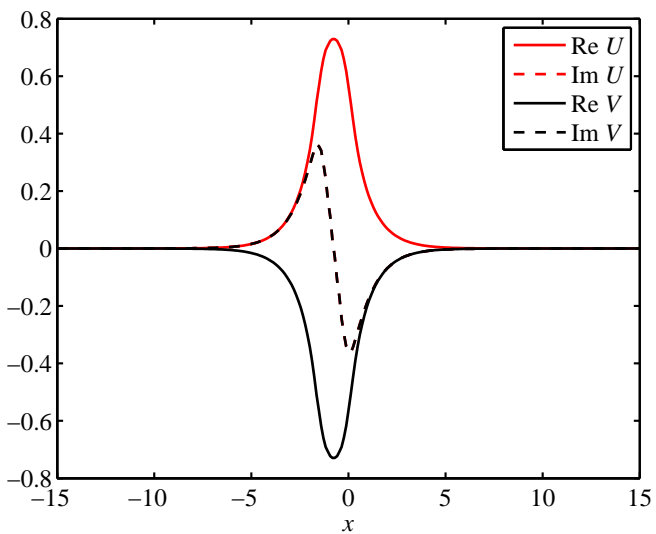

(c)

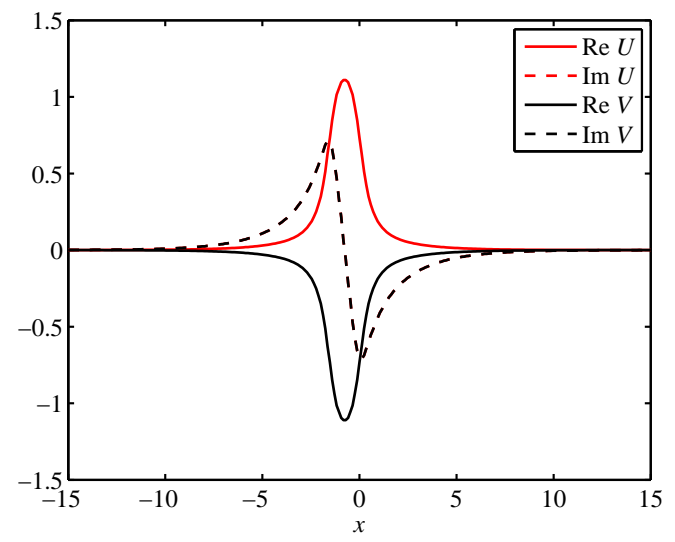

(b)

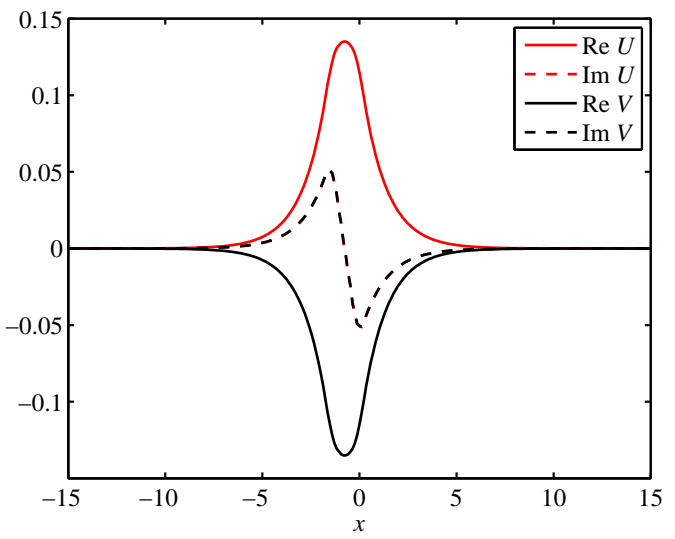

(d)

Fig. 4. (Color online) Typical examples of the nonlinear and linear defect modes in the symmetric system with $\alpha=0$ and $L=1.5$ : (a) $\omega=0.4016, \varepsilon=0.3$; (b) $\omega=-0.8715, \varepsilon=0.8$; (c) $\omega=0, \varepsilon=0.5132$; (d) $\omega=0.6099, \varepsilon=0$. Values of $\omega$ corresponding to (a) and (b) are virtually exactly predicted by Eq. (42). The linear mode in (d) is produced by Eq. (21). Because the solutions obey relation (77), the curves depicting imaginary parts of $U(x)$ and $V(x)$ are identical, while their real parts have opposite signs. 


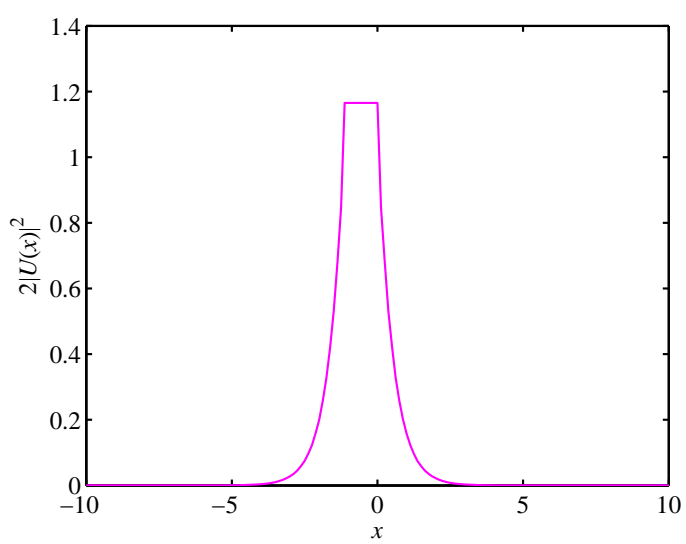

Fig. 5. (Color online) An example of the intensity profile of a numerically found stable "flat-top" soliton, at $\alpha=0, L=1.125$, $\omega=0.1$.

$$
B_{\mathrm{sol}}=\sqrt{\frac{2}{3}} e^{i \alpha / 2} \frac{\sin \theta}{\cosh (\xi \sin \theta+i \theta / 2)},
$$

where $\theta$ is related to the soliton's frequency by Eq. (12), and $\xi>0$ is an offset, which is determined by the condition of the continuity of expression (43) at $x=0$ [the continuity at $x=-L$ is provided by Eq. (44)]:

$$
\left[\cos \theta+\frac{2 \sin ^{2} \theta}{\cosh (2 \xi \sin \theta)+\cos \theta}\right] L+\frac{\alpha}{2}=2 \tan ^{-1}\left[\tanh (\xi \sin \theta) \cdot \tan \left(\frac{\theta}{2}\right)\right]+2 \pi n,
$$

with arbitrary integer $n$. The linearization limit corresponds to $\xi \rightarrow \infty$, which brings Eq. (45) back into the form of Eq. (23) for the linear DM, taking relation (12) into regard. This fact implies that the nonlinear extension of the linear DM coincides with the pinned gap solitons. In other words, it can be readily checked that, for given frequency $\omega$ belonging to bandgap (13), the nonlinear DM coincides with the respective gap soliton.

It is relevant to mention that exact composite solutions were previously obtained in other nonlinear models with multi-layer structures [55]- [57]. Most of those models are built as nonlinear lattices of the Kronig-Penney type [58].

Thus, for given $\theta \equiv \cos ^{-1}\left(\omega_{\text {sol }}\right)$, Eq. (45) determines offset $\xi$ and, through it, the entire solution given by Eqs. (43) and (44) for the fundamental pinned soliton at $n=0$, and higher-order ones at $n \neq 0$. In particular, $\xi$ and $\theta$ determine the squared amplitude of the soliton:

$$
\left|B_{\mathrm{sol}}\right|^{2}=\frac{4}{3} \frac{\sin ^{2} \theta}{\cosh (2 \xi \sin \theta)+\cos \theta} .
$$

Although the analytical solution for the pinned soliton is found here in an implicit form, it produces some simple consequences. First, the intensity profile corresponding to Eq. (43) is obviously a flat-top one, as $|U(x)|^{2}+|V(x)|^{2}=$ $2\left|B_{\text {sol }}\right|^{2}$ does not depend on $x$ in the gapless layer, see Eq. (46) and a typical example in Fig. 5 . Second, it is obvious too that the solution obeys the symmetry condition (19).

Note that the composite solution given by Eq. (43) is smoothest, i.e., without jumps of the derivative of the intensity profile, $|U(x)|^{2}$, at edges of the gapless layer, $x=0$ and $x=-L$, if Eq. (45) yields $\xi=0$. It is easy to see that, for the fundamental pinned soliton $(n=0)$, this happens at

$$
\omega=2+\alpha /(2 L) .
$$

The soliton with this frequency may exist if $\omega$ falls into bandgap (13), i.e., at $\alpha<-2 L$. Interestingly, this is precisely the case when the linear DM does not exist, pursuant to Eq. (26).

Lastly, in the limit of $L=0$, i.e., for the junction between two semi-infinite gratings with phase shift $\alpha$ between them, it is easy to find an explicit solution of Eq. (45):

$$
\xi=(2 \sin \theta)^{-1} \ln \left(\frac{\tan (\theta / 2)+\tan (\alpha / 4)}{\tan (\theta / 2)-\tan (\alpha / 4)}\right),
$$

which exists for $\theta>\alpha / 2$. Thus, solitons with internal frequency [see Eq. (12)] $\omega_{\text {sol }}=\cos \theta<\cos (\alpha / 2)$ can be pinned to the interface between two BGs with phase jump $\alpha$. The limit of $\omega_{\text {sol }}=\cos (\alpha / 2)$ exactly corresponds to the linear mode pinned to the same interface, as per Eq. (27). 


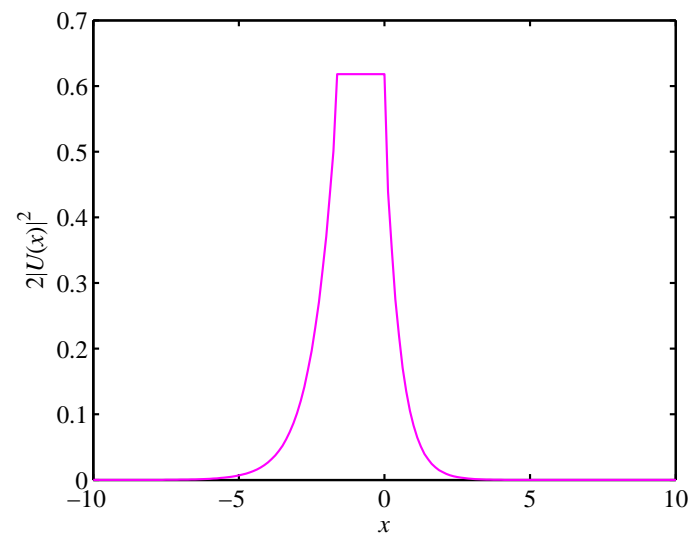

(a)

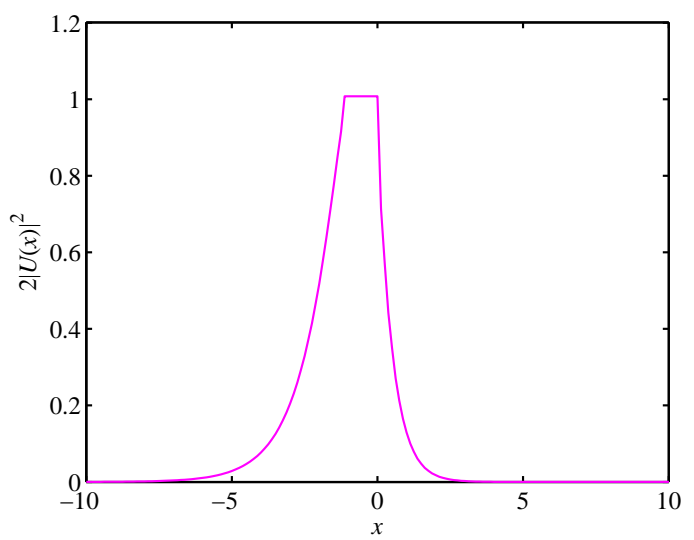

(b)

Fig. 6. (Color online) Example of intensity profiles of numerically found solitons in the asymmetric system. (a) A stable soliton for $\alpha=\pi / 4, L=1.625, \omega=0.2$. (b) An unstable soliton for $\alpha=\pi / 3, L=1.125, \omega=-0.2$

\section{D.2. The asymmetric system}

Composite flat-top solitons can be constructed for the asymmetric system too, following the pattern of ansatz (43), but it is then necessary to introduce two different offsets $\xi$ in the left and right semi-infinite gratings, which makes the composite analytical solution very cumbersome. However, the interaction of the BG soliton with the weak asymmetric interface, which is characterized by small $\alpha \ll \pi / 2$ and $L \ll \sin \theta$ in Eq. (4), can be analyzed by means of the simplest (adiabatic) version of the perturbation theory, which uses the Hamiltonian term (10), accounting for the Bragg reflectivity, to generate an effective potential of the interaction of a BG soliton with the interface, $W_{\text {int }}$, while the perturbation of the soliton's shape is disregarded [59]. A straightforward analysis yields the following asymptotic expression for the interaction force acting on the soliton with the center located at point $\Xi$ far to the left from the interface, $-\Xi \sin \theta \gg 1$ :

$$
F_{\mathrm{int}}=-\frac{\partial W_{\mathrm{int}}}{\partial \Xi} \approx \frac{8}{3}\left(\sin ^{2} \theta\right)\left(4 L \sin \theta-\alpha^{2}\right) \exp (-2(\sin \theta) \Xi)
$$

The soliton is captured by the weak interface if the the remote soliton is attracted to it, i.e., $F_{\text {int }}>0$. Thus, it follows from Eq. (49) that the capture does not occur if the soliton's amplitude is not large enough, $\sin \theta<\alpha^{2} /(4 L)$. Because $\sin \theta$ cannot exceed 1 , the final conclusion is that the interface cannot trap any BG soliton under condition

$$
L<L_{\min }^{(\mathrm{sol})}=\alpha^{2} / 4 .
$$

This prediction is qualitatively similar to that for the existence condition of the linear DM, given by Eq. (36) with $n=0$, the difference being that, for $\alpha \rightarrow 0, L_{\min }^{(\text {sol })} \sim \alpha^{2}$ is much smaller than $L_{\mathrm{min}}^{(\mathrm{DM})} \sim \alpha$.

\section{Numerical results for gap solitons}

Solitons in the symmetric system (with the phase shift $\alpha$ ) were obtained above in the implicit analytical form, based on Eqs. (43)-(45), Here, we focus on constructing a family of fundamental solitons [whose linear limit correspond to $n=0$ in Eq. [35)] in the asymmetric system in a numerical form, by means of the Newton's method. The results also include the symmetric system with $\alpha=0$ (the one with no phase shift). To this end, the exact gap soliton, given by Eq. (11) for the uniform BG, was used as the initial guess. An essential conclusion of the numerical analysis, which complies with the similar property of the symmetric system [represented by the above-mentioned correspondence between Eqs. (45) and (23), as well as between (48) and (27)], is that nonlinear DMs, obtained as a continuation of their linear counterparts given by Eqs. (21)-(35), are tantamount to gap solitons pinned to the gapless layer. Stability of the so found solitons was then tested by means of direct simulations of their perturbed evolution, which were run up to $t=10000$, using the split-step fast-Fourier-transform algorithm, with absorbers set at edges of the integration domain.

Typical examples of numerically found stable and unstable composite solitons in the asymmetric system are presented in Fig. 6. The asymmetry is clearly featured by shapes of the solitons, cf. the symmetric ones in Figs. 5. 


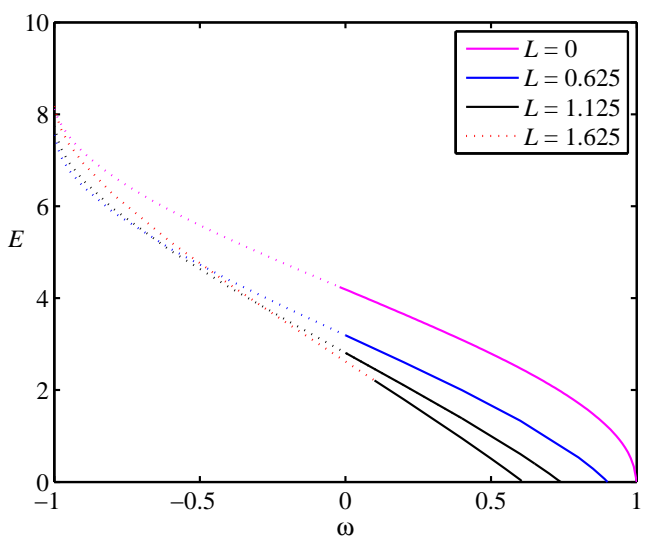

(a)

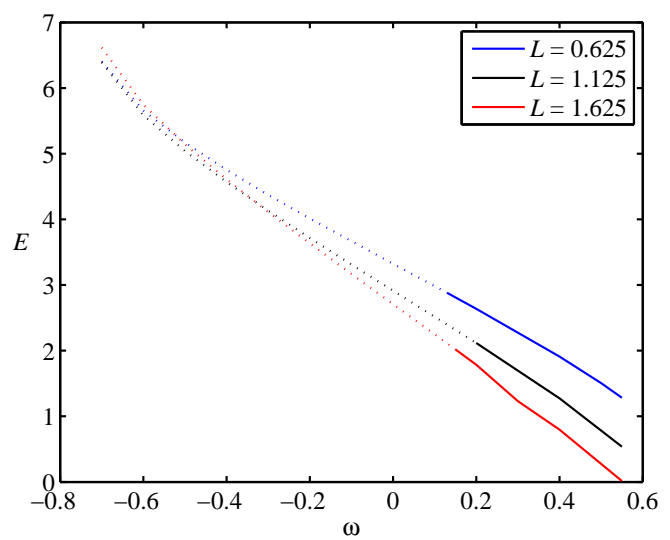

(b)

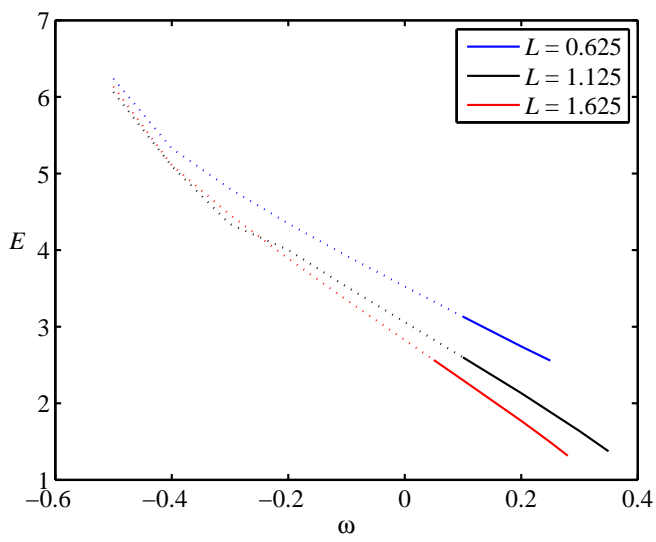

(c)

Fig. 7. (Color online) Energy $E$ of the gap solitons versus $\omega$ in the asymmetric system for different $L$. (a) $\alpha=0$; (b) $\alpha=\pi / 4$; (c) $\alpha=\pi / 3$. Continuous and dotted segments denote stable and unstable solutions, respectively. In (a), the curve for $L=0$ represents the exact solution (11), with $E=(8 / 3) \arccos \omega$, as per Eqs. (12) and (14), the boundary between the stable and unstable segments corresponding to Eq. (16). In panels (b) and (c), the numerical solution cannot be extended beyond bottom points at which the branches terminate.

Soliton families are characterized by the dependence of the total energy, $E$ [see Eq. (8)], on frequency $\omega$, which is shown in Fig. 7 for different fixed values of $L$. This figure also designates stable and unstable portions of the solution branches. Note that values of $\omega$ at $E \rightarrow 0$ in the symmetric system with $\alpha=0$ [panel[7(a)], which, obviously, correspond the linear DM, exactly agree with the respective eigenvalues (28). On the other hand, the fact that the solution branches cannot be extended up to $E=0$, i.e., to the linear limit, at $\alpha>0$, and become progressively shorter with the increase of $\alpha$ and decrease of $L$, as seen in panels $7(b, c)$, is explained by the nonexistence of the linear DM in the asymmetric system for $L$ too small, according to Eq. (36), as well as by the nonexistence of the full nonlinear solutions at small $L$, according to Eq. (50). In terms of the numerical solution, the limitation on the existence of the localized modes imposed by the asymmetry is more severe than predicted by Eq. (36), because exactly at the existence limit given by this equation, $L=\alpha /(2 \cos \alpha)$, the mode's intrinsic frequency coincides with the edge of the bandgap, i.e., the mode is still delocalized and cannot be found in the numerical form in a finite domain.

Figure 8 summarizes the numerical results by depicting stability boundaries for the solitons in the plane of $(L, E)$ for different fixed values of $\alpha$ in Eq. (4). It was found that the existence and stability areas vanish at $\alpha>\alpha_{\mathrm{cr}} \approx \pi / 2.8$, which is explained by the above-mentioned trend to the disappearance of the localization modes with the increase of asymmetry $\alpha$ [obviously, no localized modes may exist at $\alpha=\pi / 2$, when bandgap (13) with $\kappa=\cos \alpha$ shrinks to zero]. Further, in accordance with the above analytical predictions [see Eqs. (36) and (50)], gap solitons cannot be pinned by the asymmetric layer whose thickness, $L$, is too small, while there is no such limitation for the symmetric layer, with $\alpha=0$. Finally, following the pattern of the gap solitons in the uniform BG [see Eqs. (16) and (14)], the pinned solitons tend to destabilize with the increase of the energy, and they do not exist at very large values of the 


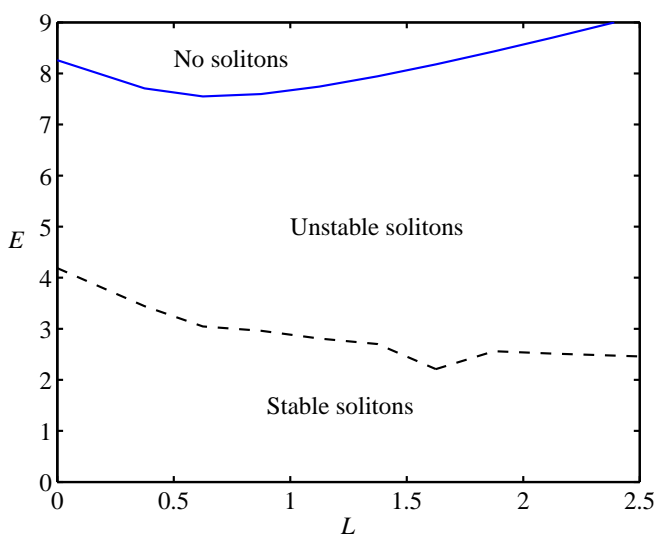

(a)

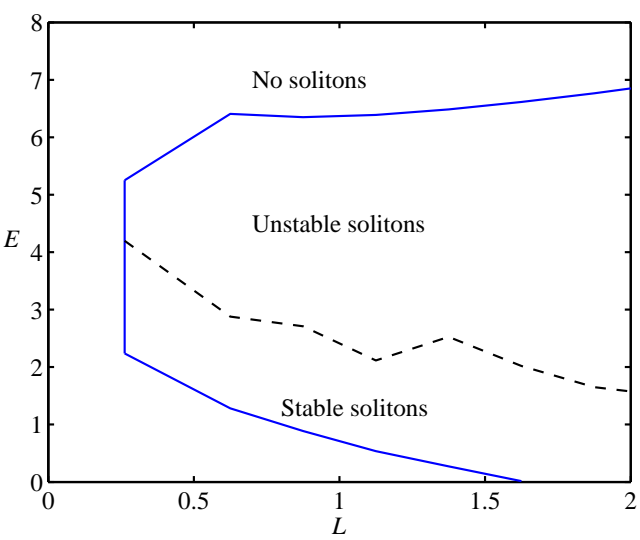

(b)

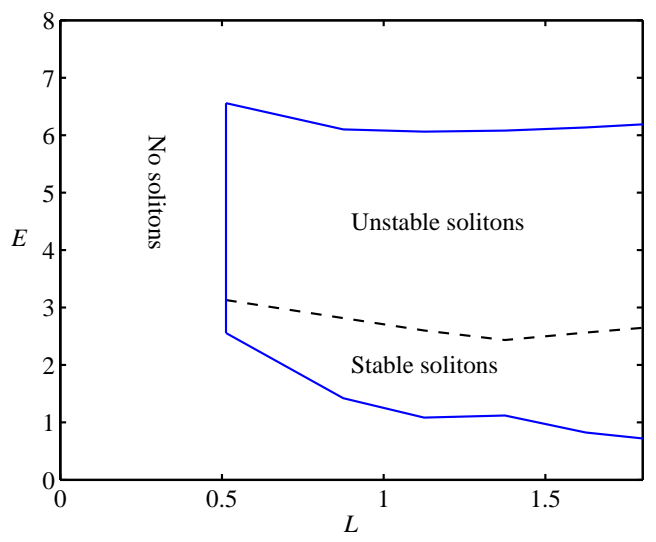

(c)

Fig. 8. (Color online) Existence and stability regions for the pinned gap solitons in the plane of $(L, E)$ of the asymmetric system at different fixed values of $\alpha$ : (a) $\alpha=0$ (the symmetric system); (b) $\alpha=\pi / 4$; (c) $\alpha=\pi / 3$. In the case of $\alpha=0$, the results for $L=0$ correspond to Eqs. (14) and (16).

energy.

Evolution of unstable solitons was investigated by means of direct simulations. As shown in Fig. 9] unstable solitons are not destroyed. Instead, they shed off a part of their energy (which is eventually eliminated by the edge absorbers) and thus transform into stable solitons with smaller energy.

\section{Collisions of moving gap solitons with the gapless layer}

Collisions of moving BG solitons with the grating-free layer is another natural problem in the present setting. It was addressed by means of direct simulations, starting with a soliton initially set far from the layer, i.e., with the center placed at some large $x_{0}>0$, and with initial velocity $c<0$, so that it travels to the left, hitting the central layer. If the layer attracts the soliton, the collision may result in its splitting into reflected and transmitted pulses, plus a trapped mode. In the uniform grating with $\kappa=1$, the initial conditions for the moving gap soliton are taken as the exact solution (written here at the initial moment, $t=0$ ) [], 9$]$ :

$$
\begin{aligned}
& u_{\mathrm{sol}}=\sqrt{\frac{2(1+c)}{3-c^{2}}}\left(1-c^{2}\right)^{1 / 4} W(X) \exp [i \phi(X)-i T \cos \theta], \\
& v_{\mathrm{sol}}=-\sqrt{\frac{2(1-c)}{3-c^{2}}}\left(1-c^{2}\right)^{1 / 4} W^{*}(X) \exp [i \phi(X)-i T \cos \theta],
\end{aligned}
$$




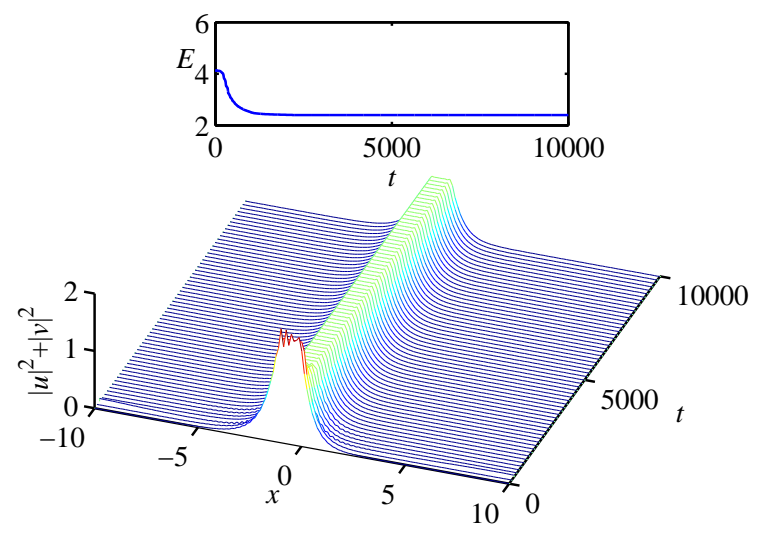

Fig. 9. (Color online) A typical example of the evolution of an unstable soliton in the asymmetric system, at $\omega=-0.3$, $L=1.125, \alpha=\pi / 4$. The top panel shows the drop of the soliton's total energy, due to radiation loss in the course of the transition to the stable shape. The eventually established soliton can be identified as one with $\omega=0.2$, which lies at the edge of the stability interval in Fig. 7(c).

$$
\begin{aligned}
X & =\left(1-c^{2}\right)^{-1 / 2} x, T=-c\left(1-c^{2}\right)^{-1 / 2} t, \\
\phi(X) & =\frac{4 c}{3-c^{2}} \tan ^{-1}\{\tanh [(\sin \theta) X] \tan (\theta / 2)\}, \\
W(X) & =(\sin \theta) \operatorname{sech}[(\sin \theta) X-i(\theta / 2)],
\end{aligned}
$$

the above solution (11) corresponding to $c=0$. In this section we report results for the soliton with $\theta=0.29 \pi$, which is stable in the uniform BG.

We first consider the layer with $L=1.0$ and $\alpha=0$ (the symmetric system). Results of the collisions with this layer are shown in Fig. 10. A slow soliton, originally moving with velocity $c=-0.1$, splits into two parts, a reflected soliton and a trapped mode. The latter one keeps $23 \%$ of the total energy of the incident soliton. The incoming soliton with $c=-0.5$ splits into three parts: a reflected one, a trapped mode, and a weak transmitted radiation jet. In this case, the trapped mode keeps $13 \%$ of the total energy. Lastly, a fast incident soliton with $c=-0.9$ splits into two parts, a transmitted one, and a weak reflected radiation jet.

As shown in Fig. 11] the difference in the case of the collision of the moving soliton with the gapless layer in the asymmetric system, with sufficiently large values of $\alpha$, is that the trapped mode does not emerge as a result. This observation is explained by the reduction and disappearance of the stability region for the pinned solitons with the growth of $\alpha$, as seen in Fig. 8 . In particular, Fig. 11] demonstrates that, at $\alpha=\pi / 3$, the slow incident soliton with $c=-0.1$ is reflected, and faster ones, with $c=-0.5$ and $c=-0.9$, are transmitted. In addition to these outcomes, radiation jets are generated too. Note that the transmission is facilitated, in this case, by the fact the incident soliton is moving from the domain with the stronger Bragg reflectivity to the one with the weaker reflectivity, according to Eq. (4).

\section{Conclusion}

We have introduced two models of the Kerr-nonlinear optical medium, which include two semi-infinite BGs (Bragg gratings), separated by the grating-free (gapless) layer of width $L$. In the symmetric model, the bandgap in both gratings is the same, while a phase shift between them, $\alpha$, is possible. The other model is asymmetric, with different widths of the bandgaps on both sides of the gapless layer, due to different Bragg reflectivities . The model can be implemented in the temporal and spatial domains alike. The attraction of the fields to the gapless layer gives rise to a DM (defect mode) in the linear version of the systems, and pinned composite gap solitons in the nonlinear one. However, in the asymmetric system the attraction competes with repulsion from the reflectivity jump, therefore the DM and pinned solitons do not exist if $L$ is too small in this system. Both the linear and nonlinear modes are constructed analytically and numerically in both systems, in exact or approximate forms. The stability region for the pinned solitons in the asymmetric system is identified by means of systematic simulations, demonstrating the shrinkage and disappearance with the increase of $\alpha$, and disappearance at small $L$ too. Collisions of freely moving solitons with the gapless layer were also studied, suggesting a possibility of the creation of the trapped mode, i.e., a pulse of standing light, as a result of the collision. 


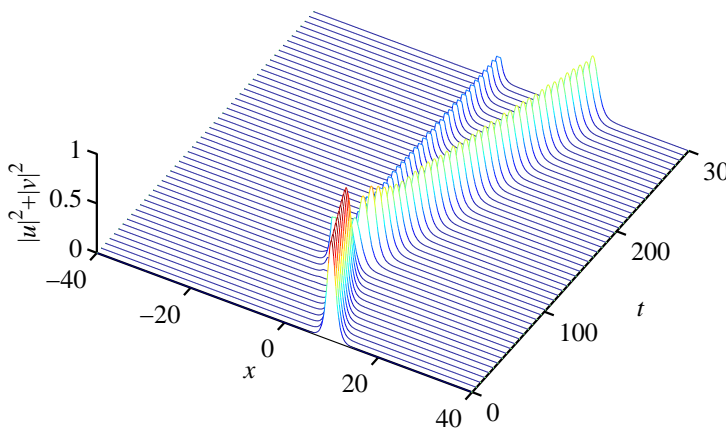

(a)

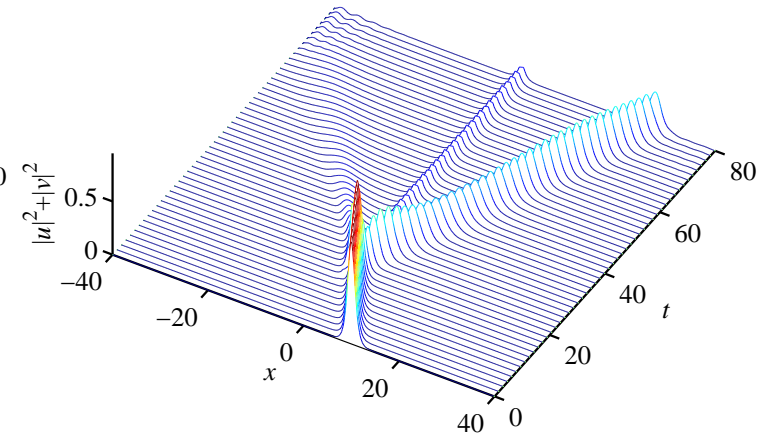

(b)

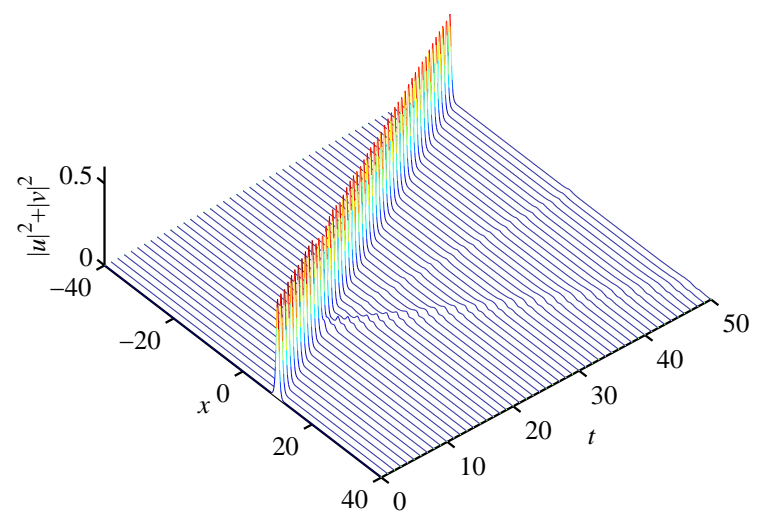

(c)

Fig. 10. (Color online) Collisions of moving gap solitons with the gapless layer in the symmetric system, with $\alpha=0$. (a) A slow gap soliton with velocity $c=-0.1$ splits into a reflected pulse and a trapped mode. (b) A gap soliton with $c=-0.5$ splits into a reflected pulse, a trapped mode, and radiation. (c) A fast gap soliton with $c=-0.9$ splits into a transmitted soliton and a radiation jet.

The work can be naturally extended in other directions. In particular, while the present analysis was focused on the fundamental trapped modes, it may be interesting to consider higher-order ones, corresponding to $n \geq 1$ in Eqs. (23), (29), (35), (36), and (45), which exist if $L$ is large enough. Another relevant extension is to address a similar model with other types of nonlinear terms, such as quadratic or resonant (two-level) ones.

\section{Acknowledgment}

This work was supported by the Thailand Research Fund through grant No. RSA5780061.

\section{References}

[1] C. R. Giles, "Lightwave applications of fiber Bragg gratings", J. Lightwave Tech. 15, 1391-1404 (1997).

[2] R. Kashyap, Fiber Bragg gratings (Academic Press: San Diego, 1999).

[3] C. M. de Sterke and J. E. Sipe, "Gap solitons," Progr. Opt. 33, 203 (1994).

[4] A. B. Aceves, "Optical gap solitons: Past, present, and future; theory and experiments", Chaos 10, 584-589 (2000).

[5] Y. S. Kivshar and G. P. Agrawal, Optical Solitons: From Fibers to Photonic Crystals (Academic Press: Boston, 2003).

[6] Yu. I. Voloshchenko, Yu. N. Ryzhov, and V. E. Sotin, "Stationary waves in nonlinear, periodically modulated media with large group retardation", Zh. Tekh. Fiz. 51, 1909-1913 (1981) [in Russian; English translation: Sov. Phys. Tech. Phys. 26, 541-544 (1981)].

[7] W. Chen and D. L. Mills, "Gap solitons and the nonlinear optical response of superlattices", Phys. Rev. Lett. 58, 160-163 (1987).

[8] A. B. Aceves and S. Wabnitz, "Self-induced transparency solitons in nonlinear refractive periodic media," Phys. Lett. A 141, 37 (1989).

[9] D. N. Christodoulides and R. I. Joseph, "Slow Bragg solitons in nonlinear periodic structures," Phys. Rev. Lett. 62, 1746 (1989). 


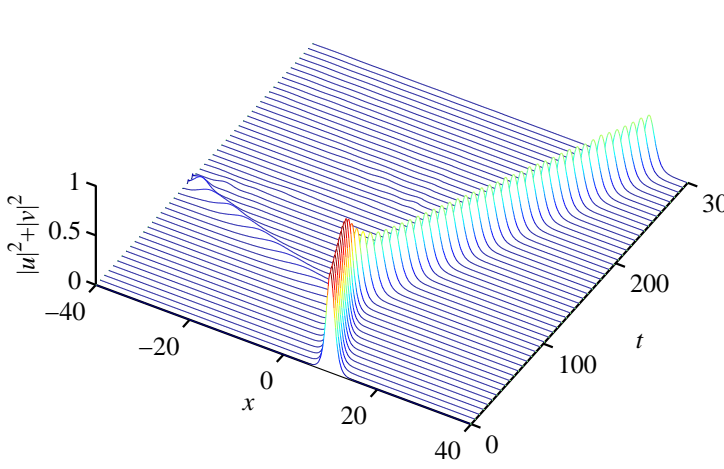

(a)

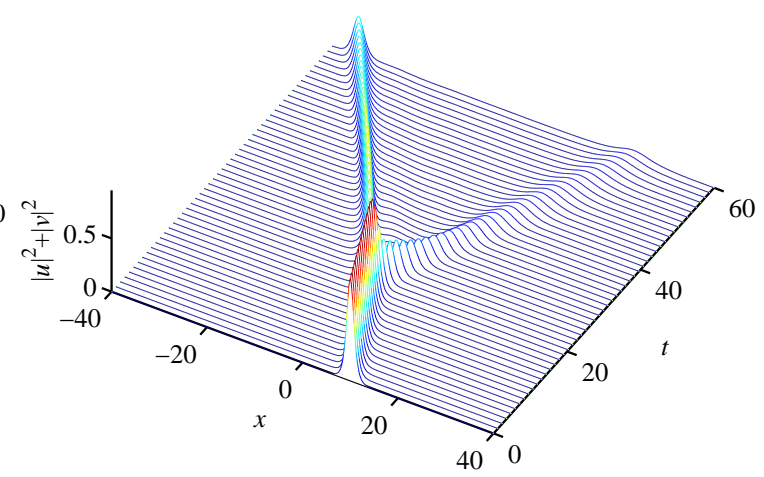

(b)

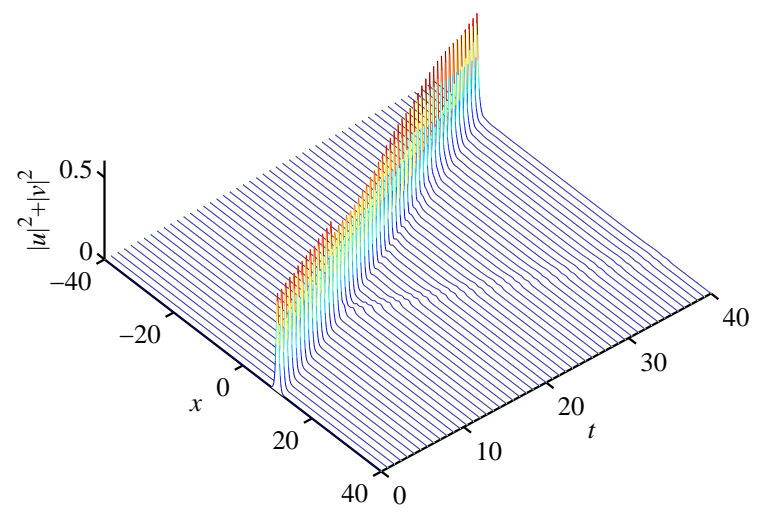

(c)

Fig. 11. (Color online) Collisions in the asymmetric system, with $L=1$ and $\alpha=\pi / 3$. (a) A slow soliton with $c=-0.1$ is reflected. (b) A soliton with $c=-0.5$ splits into a transmitted one and a reflected radiation jet. (c) A fast soliton with $c=-0.9$ is transmitted.

[10] B. J. Eggleton, R. E. Slusher, C. M. de Sterke, P. A. Krug, and J. E. Sipe, "Bragg grating solitons," Phys. Rev. Lett. 76, 1627-1630 (1996).

[11] B. J. Eggleton, C. M. de Sterke, and R. E. Slusher, "Bragg solitons in the nonlinear Schrödinger limit: Experiment and theory," J. Opt. Soc. Am. B 16, 587-599 (1999).

[12] G. Van Simaeys, S. Coen, M. Haelterman, and S. Trillo, "Observation of resonance soliton trapping due to a photoinduced gap in wave number", Phys. Rev. Lett. 92, 223902 (2004).

[13] Y. V. Kartashov, V. A. Vysloukh, and L. Torner, "Surface gap solitons", Phys. Rev. Lett. 96, 073901 (2006).

[14] Y. V. Kartashov, V. A. Vysloukh, and L. Torner, "Vector mixed-gap surface solitons", Opt. Exp. 14, 4806-4814 (2006).

[15] Y. V. Kartashov, V. A. Vysloukh, and L. Torner, "Solitons in complex optical lattices", Eur. Phys. J. - Special Topics 173, 87-105 (2009).

[16] J. T. Mok, C. M. de Sterke, I. C. M. Littler, and B. J. Eggleton, "Dispersionless slow light using gap solitons," Nature Physics 2, 775-780 (2006).

[17] J. Feng, "Alternative scheme for studying gap solitons in an infinite periodic Kerr medium," Opt. Lett. 18, 1302-1304 (1993).

[18] R. F. Nabiev, P. Yeh, and D. Botez, "Spatial gap solitons in periodic nonlinear structures," Opt. Lett. 18, 1612-1614 (1993).

[19] W. C. K. Mak, B. A. Malomed, and P. L. Chu, "Three-wave gap solitons in waveguides with quadratic nonlinearity," Phys. Rev. E 58, 6708-6722 (1998).

[20] F. Biancalana, A. Amann, and E. P. O'Reilly, "Gap solitons in spatiotemporal photonic crystals," Phys. Rev. A 77, 011801(R) (2008).

[21] T. Peschel, U. Peschel, F. Lederer and B. A. Malomed, "Solitary waves in Bragg gratings with a quadratic nonlinearity", Phys. Rev. E 55, 4730-4739 (1997).

[22] H. He and P. D. Drummond, "Ideal soliton environment using parametric band gaps", Phys. Rev. Lett. 78, 4311-4315 (1997). 
[23] C. Conti, S. Trillo, and G. Assanto, "Doubly resonant Bragg simultons via second-harmonic generation", Phys. Rev. Lett. 78, 2341-2344 (1997).

[24] J. Schöllmann, R. Scheibenzuber, A. S. Kovalev, A. P. Mayer, and A. A. Maradudin, "Stability of stationary gap solitary waves at periodically modulated surfaces", Phys. Rev. E 59, 4618 (1999).

[25] G. Kurizki, A. E. Kozhekin, T. Opatrny, and B. A. Malomed, "Optical solitons in periodic media with resonant and off-resonant nonlinearities", Progr. Optics 42, 93-146 (2001).

[26] L. Shabtay and B. A. Malomed, "Nonpolynomial Schrödinger equation for resonantly absorbing gratings", Phys. Rev. A 83, 023807 (2011).

[27] P. St. J. Russell, "Optical superlattices for modulation and deflection of light," J. Appl. Phys. 59, 3344 (1986).

[28] B. J. Eggleton. P. A. Krug, L. Poladian and F. Ouellette, "Long periodic superstructure Bragg gratings in optical fibres," Electron. Lett. 30, 1620 (1994).

[29] B. J. Eggleton, A. Ahuja, P. S. Westbrook, J. A. Rogers, P. Kuo, T. N. Nielsen, and B. Mikkelsen, "Integrated tunable fiber gratings for dispersion management in high-bit rate systems," J. Lightwave Tech. 18, 1418-1432 (2000).

[30] N. G. R. Broderick and C. M. de Sterke, "Theory of grating superstructures," Phys. Rev. E 55, 3634 (1997).

[31] J. E. Sipe, L. Poladian, and C. M. de Sterke, "Propagation through nonuniform grating structures," J. Opt. Soc. Am. A 11, 1307 (1994).

[32] T. Iizuka and C. M. de Sterke, "Corrections to coupled mode theory for deep gratings," Phys. Rev. E 61, 4491 (2000).

[33] J. B. Khurgin, "Light slowing down in Moiré fiber gratings and its implications for nonlinear optics," Phys. Rev. A 62, $013821(2000)$.

[34] R. Shimada, T. Koda, T. Ueta, and K. Ohtaka, "Strong localization of Bloch photons in dual-periodic dielectric multilayer structures," J. Appl. Phys. 90, 3905 (2001) .

[35] D. Janner, G. Galzerano, G. Della Valle, P. Laporta, S. Longhi, and M. Belmonte, "Slow light in periodic superstructure Bragg gratings," Phys. Rev. E 72, 056605 (2005)

[36] K. Levy, B. A. Malomed, "Stability and collisions of traveling solitons in Bragg-grating superstructures," J. Opt. Soc. Am. B 25, 302 (2008).

[37] T. Mayteevarunyoo and B. A. Malomed, "Gap solitons in grating superstructures", Opt. Exp. 16, 7767-7777 (2008).

[38] N. Groothoff, J. Canning, E. Buckley, K. Lyttikainen, and J. Zagari, "Bragg gratings in air-silica structured fibers," Opt. Lett. 28, 233-235 (2003).

[39] Y. N. Zhu, P. Shum, J. H. Chong, M. K. Rao, and C. Lu, "Deep-notch, ultracompact long-period grating in a large-modearea photonic crystal fiber," Opt. Lett. 28, 2467-2469 (2003).

[40] J. H. Lim, K. S. Lee, J. C. Kim, and B. H. Lee, "Tunable fiber gratings fabricated in photonic crystal fiber by use of mechanical pressure," Opt. Lett. 29, 331-333 (2004).

[41] R. H. Goodman, R. E. Slusher, and M. I. Weinstein, "Stopping light on a defect," J. Opt. Soc. Am. B 19, 1635-1652 (2002).

[42] W. C. K. Mak, B. A. Malomed, and P. L. Chu, "Interaction of a soliton with a local defect in a fiber Bragg grating", J. Opt. Soc. Am. B 20, 725-735 (2003).

[43] W. C. K. Mak, B. A. Malomed, and P. L. Chu, "Interaction of a soliton with a localized gain in a fiber Bragg grating", Phys. Rev. E 67, 0266008 (2003).

[44] R. H. Goodman and M. I. Weinstein, "Stability and instability of nonlinear defect states in the coupled mode equationsAnalytical and numerical study", Physica D 237, 2731-2760 (2008).

[45] S. Fu, Y. Liu, Y. Li, L. Song, J. Li, B. A. Malomed, and J. Zhou, "Buffering and trapping ultrashort optical pulses in concatenated Bragg gratings", Opt. Lett. 38, 5047-5050 (2013).

[46] P. Y. P. Chen, B. A. Malomed, and P. L. Chu, "Trapping Bragg solitons by a pair of defects", Phys. Rev. E 71, 066601 $(2005)$.

[47] C. M. de Sterke, I. V. Kabakova, I. Uddin, J. Jeyaratnam, and B. A. Malomed, "Spontaneous symmetry breaking in a double-defect nonlinear grating", Phys. Rev. A 88, 033825 (2013).

[48] E. N. Tsoy and C. M. de Sterke, "Propagation of nonlinear pulses in chirped fiber gratings," Phys. Rev. E 62, 2882-2890 $(2000)$.

[49] E. N. Tsoy and C. M. de Sterke, "Soliton dynamics in nonuniform fiber Bragg gratings," J. Opt. Soc. Am. B 18, 1-6 (2001).

[50] W. C. K. Mak, B. A. Malomed, and P. L. Chu, "Slowdown and splitting of gap solitons in apodized Bragg gratings," J. Mod. Opt. 51, 2141-2158 (2004).

[51] A. E. Miroshnichenko, S. Flach, and Y. S. Kivshar, "Fano resonances in nanoscale structures", Rev. Mod. Phys. 82, 2257-2298 (2010).

[52] B. A. Malomed and R. S. Tasgal, "Vibration modes of a gap soliton in a nonlinear optical medium," Phys. Rev. E 49, 5787-5796 (1994).

[53] I. V. Barashenkov, D. E. Pelinovsky, and E. V. Zemlyanaya, "Vibrations and oscillatory instabilities of gap solitons," Phys. Rev. Lett. 80, 5117 (1998).

[54] A. De Rossi, C. Conti, and S. Trillo, "Stability, multistability, and wobbling of optical gap solitons," Phys. Rev. Lett. 81, 85 (1998).

[55] Y. Kominis, "Analytical solitary wave solutions of the nonlinear Kronig-Penney model in photonic structures", Phys. Rev. E 73, 066619 (2006).

[56] Y. Kominis, A. Papadopoulos and K. Hizanidis, "Surface solitons in waveguide arrays: Analytical solutions", Opt. Exp. 15, 10041-10051 (2007). 
[57] Y. Kominis and K. Hizanidis, "Power dependent soliton location and stability in complex photonic structures", Opt. Exp. 16, 12124-12138 (2008).

[58] Y. V. Kartashov, B. A. Malomed, and L. Torner, "Solitons in nonlinear lattices", Rev. Mod. Phys. 83, 247-306 (2011).

[59] Yu. S. Kivshar and B. A. Malomed, "Dynamics of solitons in nearly integrable systems", Rev. Mod. Phys. 61, 763-915 (1989). 